\title{
Measurement of temperature rises in the femtosecond laser pulsed three- dimensional atom probe
}

\author{
A. Cerezo ${ }^{\text {a) }}$ and G. D. W. Smith \\ Department of Materials, University of Oxford, Parks Road, Oxford OX1 3PH, United Kingdom \\ P. H. Clifton \\ Oxford nanoScience, 4-6 Carters Lane, Kiln Farm, Milton Keynes MK11 3ER, United Kingdom
}

(Received 8 December 2005; accepted 2 March 2006; published online 13 April 2006)

\begin{abstract}
A previous Letter [B. Gault et al., Appl. Phys. Lett. 86, 094101 (2005)] interpreted measurements of the field evaporation enhancement under femtosecond pulsed laser irradiation of a field emitter in terms of a direct electric field enhancement by the intrinsic field of the laser light. We show that, on the contrary, the field evaporation enhancement is predominantly a thermal heating effect. Indirect measurements of the peak specimen temperature under irradiation by femtosecond laser pulses are consistent with temperature rises obtained using longer laser pulses in a range of earlier work. (C) 2006 American Institute of Physics. [DOI: 10.1063/1.2191412]
\end{abstract}

The three-dimensional atom probe (3DAP) provides chemical analysis at the ultimate level, making it possible to reconstruct the position and identity of the majority of atoms within a nanoscale volume. ${ }^{1,2}$ This instrument has provided valuable insight into the nanostructure of metallic alloys, ${ }^{3}$ and more recently of thin-film materials for information storage. ${ }^{4}$ Increasingly, atomic-scale effects in semiconductors are limiting the creation of novel electronic devices, and the 3DAP holds the potential for making significant contributions to the development of nanoelectronics.

In the 3DAP, atoms field evaporated from the apex of a needle-shaped specimen are projected onto a positionsensitive detector. ${ }^{2,3}$ Chemical identification using time-offlight mass spectrometry allows 3D reconstruction of the original elemental distributions with near-atomic resolution. Field evaporation is normally generated by a combination of a high dc voltage and nanosecond duration high voltage pulses, producing electric fields at the specimen apex of $10-50 \mathrm{~V} / \mathrm{nm}$. However, the propagation of voltage pulses requires a high degree of conductivity, limiting the application of this technique to metallic materials and highly doped semiconductors. Replacing high voltage pulsing with laser pulsing can allow atom probe analysis of less conductive materials, ${ }^{5}$ such as GaInAsP/InP multiple quantum-well structures. ${ }^{6}$ Laser pulses of 300 ps -6 ns duration have typically been used, and the enhancement of field evaporation was assumed to be principally due to specimen heating. Typical temperature rises are $200-300 \mathrm{~K}$, and only in exceptional cases is surface diffusion produced under laser irradiation in the $3 \mathrm{DAP}^{7,8}$

It has previously been suggested that where very short laser pulses are used, field evaporation may be enhanced by the intrinsic field associated with the laser light (see Ref. 2, p. 70). The intrinsic field $F$ can be calculated by equating the laser intensity $I$ with the Poynting vector, giving

\footnotetext{
${ }^{a)}$ Electronic mail: alfred.cerezo@materials.ox.ac.uk
}

$$
I=\frac{\varepsilon_{0} c}{2} F^{2}
$$

where $\varepsilon_{0}$ is the permittivity of free space and $c$ is the speed of light. In a recent Letter, Gault et al. used a femtosecond laser to enhance field evaporation from a tungsten specimen, ${ }^{9}$ and based on the observed polarization dependence, concluded that there was a direct field enhancement. However, visible light has an oscillating field of $30 \mathrm{fs}$ period. During a positive excursion of this field, an atom oscillating with a frequency of $10^{13} \mathrm{~Hz}$ would move $<0.001 \mathrm{~nm}$, a very small distance compared with interatomic spacings, before the field is reversed. It is therefore difficult to envisage how field evaporation can be enhanced by this oscillating field.

In this Letter we have used a femtosecond laser to produce enhancement of field evaporation of a tungsten specimen, in an attempt to reproduce the results from the work by Gault et al. From our detailed measurements, we show that the enhancement is due to specimen heating, and not a direct field effect. The estimated temperature rises observed in this work are consistent with the results obtained previously using longer laser pulses.

For these experiments, a diode-pumped ytterbium femtosecond laser with regenerative amplifier and second harmonic generator ( $\lambda=515 \mathrm{~nm}, 3 \mu \mathrm{J}$ per pulse) operating at pulse frequencies up to $100 \mathrm{kHz}$ was fitted to a commercial Oxford nanoScience 3DAP instrument. The laser was focused to a spot size of approximately $50 \mu \mathrm{m}$ and the polarization was controlled with a half-wave plate. In all measurements, the laser was aligned to maximize the field evaporation rate. Specimens were cooled to $60 \mathrm{~K}$ and fieldion images were obtained using $2 \times 10^{-5}$ mbar $\mathrm{He}$ (base pressure $<10^{-10}$ mbar).

Observation of field evaporation of a tungsten specimen in the field-ion image was used to measure the dependence of laser enhancement on polarization angle, as in the work by Gault et al., and the results are shown in Fig. 1. For this experiment the laser was operated at a repetition frequency of $100 \mathrm{kHz}$ and a pulse energy of approximately $0.5 \mu \mathrm{J}$ (equivalent to a voltage pulse fraction of $15 \%$, see below). At each polarization, the dc voltages required to generate field 


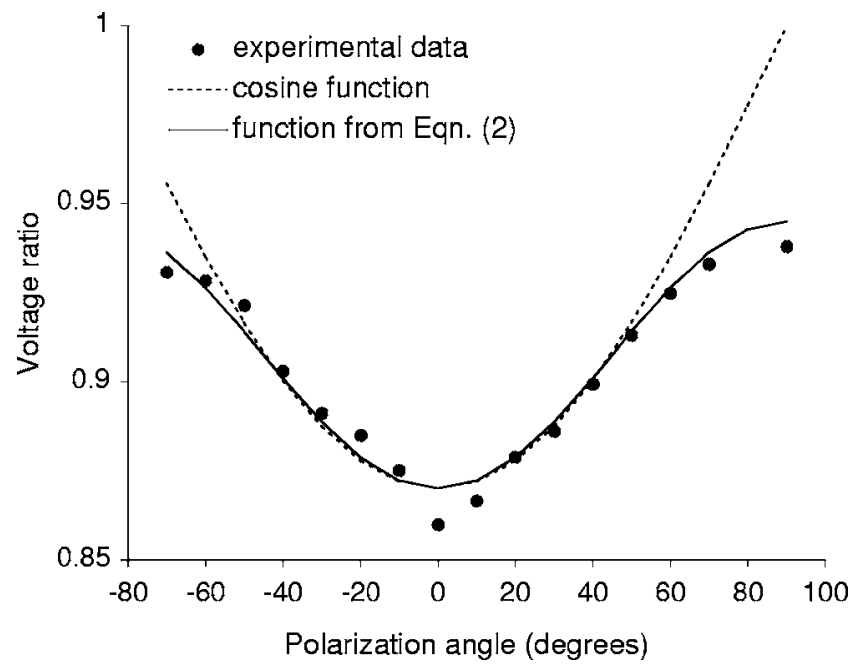

FIG. 1. Dependence of the voltage ratio (dc voltage required for laserassisted field evaporation as a ratio of that needed for field evaporation without laser irradiation) as a function of the angle of polarization of the laser light relative to the specimen axis. Also shown are a cosine function, as would be expected for direct field enhancement, and the function of Eq. (2) based on specimen heating effects.

evaporation of $0.01-0.02$ layer/s were recorded both with and without laser irradiation of the specimen, and the ratio of the two voltages was used to give an estimate of the laser enhancement of field evaporation. The enhancement was clearly strongly polarization dependent, as reported by Gault et al., with light polarized parallel to the specimen axis having a stronger effect than perpendicularly polarized light. However, our results differ in two important respects. Firstly, the amplitude of the cosine function which fits the data (Fig. 1 ) is less than that seen in the results of Gault et al. Secondly, we observe laser enhancement even for perpendicularly polarized light, indicating that the assumption of direct field enhancement is not correct.

3DAP analysis was used to determine the variation of laser enhancement with intensity, which should follow a square-root dependence if Eq. (1) applies. Short blocks of data were acquired using laser pulsing at $1 \mathrm{kHz}$ repetition rate with different settings of pulse energy. From the results, the laser enhancement (relative reduction in the voltage required for field evaporation) was calculated as a function of pulse energy (Fig. 2). Instead of a square-root variation, the data fit a linear dependence at low powers, suggesting that the main mechanism of field evaporation enhancement is specimen heating. Data acquired previously for the dependence of pulsed evaporation field with temperature for tungsten indicated a reduction of $10 \%$ in evaporation field for a temperature rise of $200 \mathrm{~K}^{7}{ }^{7}$ This value was used to obtain an estimate of the temperature rises being generated under the femtosecond laser irradiation, given on the right-hand axis of Fig. 2. Whereas for normal conditions (15\% laser enhancement) the temperature rises are limited to $300 \mathrm{~K}$, under higher laser pulse energies temperatures of up to $1000 \mathrm{~K}$ can be reached.

During the imaging experiments, it was observed that at low laser pulse energies, the pattern of field evaporation was similar to that using voltage pulses. However, at higher pulse energies (above $1.5 \mu \mathrm{J}$ ) surface diffusion was clearly observed. Observation of surface diffusion clearly shows that significant thermal effects are occurring, and together with

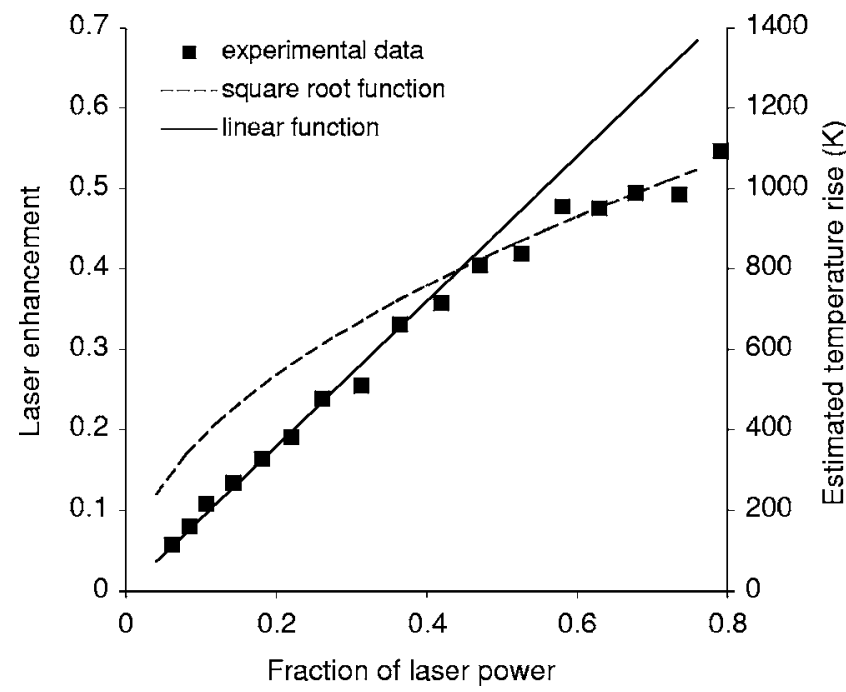

FIG. 2. Reduction in the voltage required for field evaporation under laser illumination, as a function of pulse energy for a spot size of $50 \mu \mathrm{m}$. The data do not follow a square-root dependence, as would be expected for a direct field enhancement effect, but are closer to a linear dependence at low powers, with a drop-off at higher powers.

the other evidence, leads us to conclude that the effect of the laser is predominantly one of specimen heating. This is further supported by the consistency of the estimates of temperature rise based on the assumption of a purely thermal effect. The point at which surface diffusion starts is where the data in Fig. 2 depart from the linear: the deviation from a linear dependence is probably due to this surface diffusion. At this point, the estimated temperature rise is $800 \mathrm{~K}$, which agrees well with the estimate by Kellogg of $825 \mathrm{~K}$ for the temperature where a small amount of surface diffusion was observed. $^{7}$

If the effect is thermal, the question of why there is a strong polarization dependence of field evaporation enhancement remains. Robins et al. calculated that there is a strong diffraction effect for light incident on a small cylinder. ${ }^{10}$ For a cylinder radius of $(0.05-0.1) \lambda$, light polarized parallel to the axis of the cylinder is absorbed 4.8-1.5 times more strongly than perpendicularly polarized light. Plots of polarization dependence should therefore be interpreted as the sum of heating effects from the two polarization components, i.e.,

$$
k_{1} \cos ^{2} \theta-k_{2} \sin ^{2} \theta,
$$

where $k_{1}$ and $k_{2}$ are related to the absorption coefficients. For the data shown in Fig. 1, a good fit is obtained using a ratio of $k_{1} / k_{2}=2.4$. This is smaller than would be expected from the calculations of Robins et al. ${ }^{10}$ assuming a tip radius of $20 \mathrm{~nm}$. However, given the rapid change in the calculated ratio for a small change in size, together with uncertainties in exactly how the calculation applies to a needle-shaped specimen, there is reasonable agreement. Gault et al. used a longer wavelength $(800 \mathrm{~nm})$, leading to an even more pronounced absorption of the parallel polarization component. Thus, it is not surprising that they measured no enhancement at all using perpendicularly polarized light. Since they had no reference point from which to construct a fit to the data, it was easy to fit a $\cos \theta$ curve over the range of angles for which they had measurements, as seen in Fig. 1, even though the true dependence is $\cos ^{2} \theta$.

to AIP license or copyright; see http://apl.aip.org/apl/copyright.jsp 
It is often stated that femtosecond lasers produce no heating. This may be the case in laser machining, where the energy deposited by the laser is removed in the ablated material. With the much lower pulse energies in the laser pulsed 3DAP, material is not lost through ablation, and so the energy deposited into the specimen must, finally, produce lattice heating. However, contrary to the view expressed by Gault $e t$ al., the temperature rises under normal pulsed laser atom probe operation are low and spatial resolution is not degraded. ${ }^{8}$ Temperature rises estimated for the field evaporation of tungsten under normal conditions are approximately $300 \mathrm{~K}$, where no surface diffusion occurs until $800 \mathrm{~K}$.
${ }^{1}$ A. Cerezo, T. J. Godfrey, and G. D. W. Smith, Rev. Sci. Instrum. 59, 862 (1988).

${ }^{2}$ M. K. Miller, A. Cerezo, M. G. Hetherington, and G. D. W. Smith, Atom Probe Field Ion Microscopy (Oxford University Press, 1996).

${ }^{3}$ M. K. Miller, Atom Probe Tomography (Plenum, 2000).

${ }^{4}$ D. J. Larson, A. K. Petford-Long, Y. Q. Ma, and A. Cerezo, Acta Mater. 52, 2847 (2004).

${ }^{5}$ G. L. Kellogg and T. T. Tsong, J. Appl. Phys. 51, 1184 (1980).

${ }^{6}$ J. A. Liddle, A. Norman, A. Cerezo, and C. R. M. Grovenor, Appl. Phys. Lett. 54, 1555 (1989).

${ }^{7}$ G. L. Kellogg, J. Appl. Phys. 52, 5320 (1981).

${ }^{8}$ A. Cerezo, C. R. M. Grovenor, and G. D. W. Smith, J. Microsc. 141, 155 (1986).

${ }^{9}$ B. Gault, F. Vurpillot, A. Bostel, A. Menand, and B. Deconihout, Appl. Phys. Lett. 86, 094101 (2005).

${ }^{10}$ E. S. Robins, M. J. G. Lee, and P. Langlois, Can. J. Phys. 64, 111 (1986). 